\title{
Prompt emission spectra from the photosphere of a GRB
}

\author{
D. Giannios
}

\author{
Max Planck Institute for Astrophysics, Box 1317, 85741 Garching, Germany \\ e-mail: giannios@mpa-garching.mpg.de
}

Received 10 February 2006 / Accepted 10 July 2006

ABSTRACT

\begin{abstract}
I explore the observational appearance of the photosphere of an ultrarelativistic flow with internal dissipation of energy ("dissipative" GRB model). As a case study, I use the magnetic reconnection model (AC model) that makes robust predictions on the energy dissipation rates at different radii in the flow. With analytical and numerical tools for the radiative transfer problem, I show that the flow develops a hot photosphere where inverse Compton scattering leads to highly non-thermal spectrum. For a wide range of luminosities and baryon loadings of the flow, this spectrum is very close to the observed prompt GRB emission. Its luminosity ranges from $\sim 3$ to $20 \%$ of that of the total energy input.
\end{abstract}

Key words. gamma rays: bursts - radiation mechanisms: general - methods: statistical

\section{Introduction}

Although much progress has been made the recent years in our understanding the central engine and of the afterglow emission of Gamma-ray bursts (GRBs), much less is known about the physical processes responsible for the prompt emission. Theoretical arguments related to the so called compactness problem (e.g. Piran 1999) suggest that the emitting material is ultrarelativistic with bulk Lorentz factors $\Gamma \gtrsim 100$. The typical observed GRB spectrum has a characteristic peak (in $E \cdot f(E)$ representation) in the sub-MeV range that is smoothly connected to low and high frequency power-laws, usually modeled with the Band spectrum (Band et al. 1993; Preece et al. 1998). Models for the prompt GRB phase have to account for both the acceleration of the flow and its observed spectral properties.

For a flow to be accelerated to high bulk Lorentz factors, it must start with high energy-to-rest-mass ratio. Depending on whether the energy is in thermal or magnetic form, one has a fireball (Paczynski 1986; Goodmann 1986) or a Poynting-flux dominated flow (Thompson 1994; Mészáros \& Rees 1997; Spruit et al. 2001; Drenkhahn \& Spruit 2002; Lyutikov \& Blandford 2003). In the fireball model, the flow passes through an initial phase of rapid acceleration where $\Gamma \sim r$ until most of the internal energy has been used to accelerate the flow (unless the baryon loading is very low and radiation decouples from matter before the acceleration phase is over). Further out, internal shocks (Rees \& Mészáros 1994; Sari \& Piran 1997) can dissipate part of the kinetic energy and power the prompt emission. In magnetic models for GRBs, the flow acceleration is more gradual (e.g. Drenkhahn \& Spruit 2002; Vlahakis \& Königl 2003) and dissipation of magnetic energy through magnetic reconnection (Drenkhahn 2002) or current driven instabilities (Lyutikov \& Blandford 2003; Giannios \& Spruit 2006) can directly power the prompt emission.

If the dissipated energy leads to fast particles, then the synchrotron or synchrotron self Compton mechanism appears as a natural one for the prompt emission. On the other hand, the large number of bursts with low energy slopes steeper than the synchrotron model predictions (e.g. Crider et al. 1997;
Frontera et al. 2000; Girlanda et al. 2003), have triggered alternative suggestions for the origin of the GRB emission. These include saturated Comptonization (Liang 1997), Comptonization by thermal electrons (Ghisellini \& Celotti 1999) and the photospheric emission of the flow (Mészáros \& Rees 2000; Ryde 2004).

Both fireball and magnetic models predict some degree of photospheric emission that comes from the region where the flow becomes optically thin to Thomson scattering. If no energy dissipation takes place in the photospheric region, this emission is expected to be quasi-thermal and its peak should be clearly observed in the case of fireball models (Daigne \& Mochkovitch 2002); which is not the case. On the other hand, if there is substantial energy dissipation (through internal shocks, magnetic reconnection or MHD instabilities) close to the photospheric region, the emitted spectrum can strongly deviate from a black body (Mészáros \& Rees 2000; Pe'er et al. 2005, 2006; Ramirez-Ruiz 2005).

In this work, I study the spectrum of the photospheric emission in dissipative GRB models. I use the magnetic reconnection model (Drenkhahn 2002; Drenkhahn \& Spruit 2002) since it makes robust predictions for the characteristics of the flow and the rate of magnetic energy dissipation at different radii and study in detail the radiative transfer in this model. Similar considerations can be applied to other dissipative models (see also Pe'er et al. 2006).

The study shows that while deep inside the flow radiation and matter are in approximate thermodynamic equilibrium, direct heating of the electrons through energy dissipation leads to increase of their temperature as a function radius at radii where the flow is still thick to Compton scattering (about a factor of ten below the photospheric radius).

A Monte Carlo code has been implemented to study the effect of Compton scattering at the photospheric region. The electron temperature is determined by balancing the heating by dissipation with the cooling by Comptonization. The calculations show that the flow develops a hot photosphere with comoving electron temperatures of the order of $\sim 30 \mathrm{keV}$ (and larger 
further out) for a large range of luminosities and baryon loadings of the flow. Inverse Compton scattering of the underlying thermal radiation leads to a Band-like spectrum which peaks at $\sim 1 \mathrm{MeV}$ (in the central engine frame) followed by a nearly flat high energy spectrum (with $E \cdot f(E) \sim E^{0}$ ) that extends up to a few hundred $\mathrm{MeV}$.

In the next section, I discuss various dissipative models for GRB outflows and review the main features of the magnetic reconnection model. Analytical estimates for the radiative transfer and the electron temperature in the photospheric region are given in Sect. 3. The description of the Monte Carlo code and the numerical results are presented in Sect. 4. The last two sections contain the discussion and the conclusions.

\section{Dissipation close to the photosphere}

The fireball model for GRB outflows (Paczyński 1986; Goodman 1986) predicts the emission of a photospheric component from the region where radiation and matter decouple. This decoupling takes place close to the Thomson photosphere of the flow. The photospheric radiation can carry away most of the luminosity of the flow (if the latter is still in its acceleration phase when the decoupling takes place) or a smaller fraction $\sim\left(r_{\mathrm{s}} / r_{\mathrm{ph}}\right)^{2 / 3}$ of it depending on the baryon loading of the flow. Here $r_{\mathrm{s}}$ stands for the saturation radius where the flow reaches its terminal Lorentz factor and $r_{\mathrm{ph}}$ is the photospheric radius. In the absence of energy dissipation around $r_{\mathrm{ph}}$, the photospheric component is expected to be quasi-thermal. Although a small fraction of GRBs do show thermal emission (Ryde 2004), the typical prompt GRB spectrum cannot be fitted by a black body.

Internal shocks (Rees \& Mészáros 1994) in an unsteady flow are usually invoked to dissipate a fraction of the bulk kinetic energy of the flow into fast particles and random magnetic fields and to power the prompt emission. For a range of the parameter space of the internal shock model, these shocks can take place below the Thomson photosphere, influencing the strength and the spectrum of the photospheric component (Rees \& Mészáros 2000, 2005; Pe'er et al. 2005, 2006).

In the case of Poynting-flux dominated flows, both acceleration of the flow and emission of radiation can take place through dissipation of magnetic energy. Magnetic energy can be dissipated directly through reconnection in a flow where the magnetic field changes polarity on small scales (AC flow; Drenkhahn 2002; Drenkhahn \& Spruit 2002) or via MHD instabilities in an axisymmetric flow (Giannios \& Spruit 2006). The magnetic dissipation and acceleration in these models is gradual and takes place over several decades in radius that typically include the photospheric radius. The photospheric component can be quite strong, of the order of $\$ 20 \%$ of the luminosity of the flow.

Here, I focus on the observational appearance of the photospheric component in an "AC" flow. The basic features of the dynamics of the flow are reviewed in the next section, while the detailed dynamical calculations are presented in Drenkhahn (2002) and Drenkhahn \& Spruit (2002).

\subsection{The reconnection or "AC" model}

An important physical quantity of the flow in the "AC" model is the ratio $\sigma_{0}$ of the Poynting flux to kinetic energy flux at the Alfvén point $r_{0}$. This is a quantity that plays similar role to the "baryon loading" parameter that appears in fireball models. the ratio $\sigma_{0}$ determines the terminal bulk Lorentz factor of the flow $\Gamma_{\infty}$ which is of the order of $\sim \sigma_{0}^{3 / 2}$. The flow must start Poynting-flux dominated with $\sigma_{0} \gtrsim 30$ for it to be accelerated to ultrarelativistic speeds with $\Gamma_{\infty} \gtrsim 100$.

In an "AC" model, such as produced by an inclined rotator (Coroniti 1990), the magnetic field in the flow changes polarity on small scale $\lambda$ of the order of the light cylinder in the central engine frame (i.e. $\lambda \simeq 2 \pi c / \Omega$, where $\Omega$ is the angular frequency of the rotator). In the flow, the energy density of the magnetic field is larger than the rest mass energy density and the reconnection speed is increased by the relativistic kinematics to subrelativistic speeds (Lyutikov \& Uzdensky 2003; Lyubarsky 2005). Magnetic reconnection is modeled to proceed at a fraction $\varepsilon \sim 0.1$ of the Alfvén speed (which is essentially the speed of light for a magnetically dominated flow). Because of dissipation of the magnetic energy, about half of the Poynting flux converts into kinetic flux (i.e. acceleration of the flow) and the other half into internal energy of the flow. The dissipation is gradual and takes place up to the "saturation radius" $r_{\mathrm{s}}$ where reconnection stops and the flow achieves its terminal Lorentz factor.

Under the assumption of $1 \mathrm{D}$, steady flow, the relativistic MHD equations can be solved analytically for radii $r_{0} \ll r \ll$ $r_{\mathrm{s}}$, yielding the following self similar scalings for the comoving number density and the comoving magnetic field strength (Drenkhahn 2002)

$$
\begin{aligned}
& n^{\prime}=\frac{1.5 \times 10^{17}}{r_{11}^{7 / 3}} \frac{L_{52}}{(\varepsilon \Omega)_{3}^{1 / 3} \sigma_{0,2}^{2}} \mathrm{~cm}^{-3} \\
& B^{\prime}=\frac{1.4 \times 10^{8}}{r_{11}^{4 / 3}} \frac{L_{52}^{1 / 2}}{(\varepsilon \Omega)_{3}^{1 / 3} \sigma_{0,2}^{1 / 2}} \text { Gauss }
\end{aligned}
$$

respectively. The notation $A=10^{x} A_{x}$ is used and the "reference values" of the model parameters are $\sigma_{0}=100, \varepsilon=0.1, \Omega=$ $10^{4} \mathrm{rad} \mathrm{s}^{-1}, L=10^{52} \mathrm{erg} \mathrm{s}^{-1}$ sterad $^{-1}$; very close to those used in previous studies of the model (see Drenkhahn 2002; Drenkhahn \& Spruit 2002; Giannios \& Spruit 2005).

The model predicts gradual acceleration of the flow with $\Gamma \sim$ $r^{1 / 3}$ in the regime $r_{0} \ll r \ll r_{\mathrm{s}}$, while no further acceleration takes place above the saturation radius. The bulk Lorentz factor of the flow is, thus, approximately given by the expression

$$
\begin{aligned}
& \Gamma=\Gamma_{\infty}\left(\frac{r}{r_{\mathrm{s}}}\right)^{1 / 3}=148 r_{11}^{1 / 3}(\varepsilon \Omega)_{3}^{1 / 3} \sigma_{0,2}^{1 / 2}, \text { for } r<r_{\mathrm{s}} \\
& \Gamma=\Gamma_{\infty}=\sigma_{0}^{3 / 2}, \quad \text { for } \quad r \geq r_{\mathrm{s}} .
\end{aligned}
$$

The saturation radius is given by

$r_{\mathrm{s}, 11}=\frac{\pi c \Gamma_{\infty}^{2}}{3 \varepsilon \Omega}=310 \frac{\sigma_{0,2}^{3}}{(\varepsilon \Omega)_{3}}$

Another characteristic radius of the flow is the Thomson photosphere. The optical depth between two points in the flow can be found by integrating the expression $\mathrm{d} \tau=\Gamma(1-\beta \cos \theta) n^{\prime} \sigma_{\mathrm{T}} \mathrm{d} s$ (Abramowicz et al. 1991), where $\beta=v / c$ and $\theta$ the angle of a photon path with respect to the radial direction. Integrating the previous expression from $r$ to $\infty$ for a radially moving photon, one gets the characteristic Thomson optical depth as a function of radius

$\tau=\frac{20}{r_{11}^{5 / 3}} \frac{L_{52}}{(\varepsilon \Omega)_{3}^{2 / 3} \sigma_{0,2}^{5 / 2}}$. 
The location of the photosphere is found by setting $\tau=1$ and solving for $r$

$r_{\mathrm{ph}, 11}=6 \frac{L_{52}^{3 / 5}}{(\varepsilon \Omega)_{3}^{2 / 5} \sigma_{0,2}^{3 / 2}}$.

The location of the photospheric radius with respect to the saturation one determines the nature of the emitted spectra expected from the flow. If, for example, $r_{\mathrm{ph}} \gg r_{\mathrm{s}}$, all the energy dissipation takes place in optically thick conditions and the radiation is efficiently thermalized. On the other hand, if $r_{\mathrm{s}} \gtrsim r_{\mathrm{ph}}$, energy dissipation at moderate and low optical depths leads to a photospheric emission that has a highly non-thermal appearance that can be directly related with the characteristic GRB emission. These points become clear in the next sections where the radiative transfer around the region of the photosphere is studied with analytical and numerical tools.

\section{Analytical estimates}

Energy dissipation in an "AC" flow is gradual and takes place over many decades of radii up to the saturation radius $r_{\mathrm{s}}$. Half of the dissipated Poynting flux serves to accelerate the flow, while the other half is released as internal energy in the flow. The rate of energy density release in a comoving frame can be found by the following considerations. The time scale over which the magnetic field decays is that of advection of magnetic field of opposite polarity to the reconnection area. The reconnection speed is $v_{r}=\varepsilon v_{\mathrm{A}} \simeq \varepsilon c$, while the magnetic field changes polarity over a length scale $\lambda^{\prime}=2 \pi \Gamma c /(\varepsilon \Omega)$ measured in a frame comoving with the flow. The decay timescale for the magnetic field, therefore, is

$t_{\mathrm{dec}}=\frac{\lambda^{\prime}}{v_{r}}=\frac{2 \pi \Gamma}{\varepsilon \Omega}$.

Using the last expression and Eqs. (2) and (4), the rate of magnetic energy density dissipation in the comoving frame is

$P_{\text {diss }}=\frac{B^{\prime 2} / 8 \pi}{t_{\text {dec }} / 2}=\frac{1.5 \times 10^{15}}{r_{11}^{3}} \frac{L_{52}}{\sigma_{0,2}^{3 / 2}} \quad \mathrm{erg} \mathrm{cm}^{-3} \mathrm{~s}^{-1}$.

The fate of the released energy is rather uncertain. An interesting possibility is that dissipation leads to MHD turbulence where particle acceleration can take place by scattering of photons by Alfvén waves at the $\tau \sim 1$ region of the flow (Thompson 1994). On the other hand, the magnetic energy can directly be dissipated to the particles in the flow, most likely to the electrons due to their higher mobility. Here I assume that a fraction $f_{\mathrm{e}}$ of order unity of the energy is heating the electrons of the flow. The electrons are assumed to have a thermal distribution. Deep in the flow, the relaxation timescale of the electrons due to Coulomb collisions is fast enough to ensure thermalization. I return to this issue in Sect. 4.3.

Deep inside the flow the released energy is efficiently thermalized and shared between particles and radiation. Assuming complete thermalization, integration of the energy released at different radii in the flow, taking into account adiabatic cooling, leads to the following expression for the comoving temperature of the flow (Giannios \& Spruit 2005)

$T_{\text {th }}=\frac{0.7}{r_{11}^{7 / 12}} \frac{L_{52}^{1 / 4}}{(\varepsilon \Omega)_{3}^{1 / 12} \sigma_{0,2}^{1 / 2}} \mathrm{keV}$.
Comoving temperatures of a few hundred $\mathrm{eV}$ are typical at the region where the optical depth of the flow is of order of unity (see the last expression and Eq. (6)). At these temperatures the Compton scattering cross section dominates over free-free absorption so that deviation from thermal spectra is expected. It turns out, however, that the assumption of equilibrium of radiation and particles breaks down deeper in the flow and that the "photospheric" spectra are highly non-thermal for typical GRB parameters.

The last point becomes clear if one looks at the energy balance of the electrons in the flow. The electrons are heated by magnetic energy dissipation and cool mainly through radiative cooling (the adiabatic cooling and the energy exchange with the protons are much slower). An obvious candidate for radiative cooling of the electrons in a strongly magnetized flow is synchrotron cooling since the energy density of the magnetic field is larger than that of radiation

$\frac{U_{\mathrm{B}}}{U_{r}}=\frac{B^{\prime 2} / 8 \pi}{a T_{\mathrm{th}}^{4}}=23 \frac{\sigma_{0,2}}{r_{11}^{1 / 3}(\varepsilon \Omega)_{3}^{1 / 3}}$.

This suggests dominance of synchrotron over Compton cooling if the flow is optically thin to synchrotron emission. It turns out that this is not the case in the photospheric region under consideration and that synchrotron radiation is strongly self-absorbed (I return to this issue in the end of this section). The dominant cooling process for the electrons is, thus, Compton cooling.

The Compton cooling rate for the electrons is given by the expression (e.g. Rybicki \& Lightman 1979)

$P_{\text {Comp }}=4 n^{\prime} \Theta_{\mathrm{e}} c \sigma_{\mathrm{T}} U_{r}$,

where $\Theta_{\mathrm{e}}=K_{B} T_{\mathrm{e}} /\left(m_{\mathrm{e}} c^{2}\right)$ and $\sigma_{\mathrm{T}}$ is the Thomson scattering cross section. Equating the heating rate (given by Eq. (8)) to the cooling rate of the electrons, I solve for the electron temperature

$T_{\mathrm{e}}=2.0 r_{11}^{5 / 3} f_{\mathrm{e}, 1} \frac{(\varepsilon \Omega)_{3}^{2 / 3} \sigma_{0,2}^{5 / 2}}{L_{52}} \mathrm{keV}$,

where $f_{\mathrm{e}, 1}$ stands for $f_{\mathrm{e}}=1$. So the electron temperature increases as a function of radius and equilibrium of the electrons and radiation is reached deep in the flow at the radius where $T_{\text {th }}=T_{\mathrm{e}}$. The last equation defines a characteristic radius which I call the equilibrium radius

$r_{\mathrm{eq}, 11}=0.6 \frac{L_{52}^{5 / 9}}{f_{\mathrm{e}, 1}^{4 / 9}(\varepsilon \Omega)_{3}^{1 / 3} \sigma_{0,2}^{4 / 3}}$,

where Eqs. (9) and (12) have been used to derive the last expression. Note that equilibrium of matter and radiation is achieved at a radius that is factor of ten shorter than that of the Thomson photosphere, or at an optical depth (combining Eqs. (5) and (13))

$\tau_{\mathrm{eq}}=46 \frac{f_{\mathrm{e}, 1}^{20 / 27} L_{52}^{2 / 27}}{(\varepsilon \Omega)_{3}^{1 / 9} \sigma_{0,2}^{5 / 18}}$

which depends weakly on the model parameters and is much larger than unity.

At radii $r>r_{\text {eq }}$, the electron temperature is higher than that of the radiation field and upscattering of the photons takes place. Because of the increase of the electron temperature with radius, this upscattering becomes more efficient close to the location of the photosphere. The electron temperature there can be found by combining Eqs. (6) and (12) which give $T_{\mathrm{e}, \mathrm{ph}}=40 f_{\mathrm{e}, 1} \mathrm{keV}$ 
independently of the parameters of the model except to the fraction $f_{\mathrm{e}}$. For temperatures $T_{\mathrm{e}} \gtrsim 40 \mathrm{keV}$ in the $\tau_{\mathrm{T}} \lesssim 1$ region, the characteristic signature of unsaturated Comptonization is expected to lead to spectra with hard non-thermal appearance.

The previous estimates are based on the assumption that synchrotron emission in the flow is strongly self-absorbed and therefore the electrons cool mainly through Compton upscattering the radiation field. I can now check the validity of this assumption by estimating the synchrotron (and free-free) absorption optical depths in the flow and the characteristic turn-over frequency below which radiation becomes optically thick. The absorption optical depth for a photon traveling from radius $r$ to $2 r$ is given by the expression $\tau_{v}=\alpha_{v} r / \Gamma$, where $\alpha_{v}$ is the absorption coefficient as measured by the comoving observer. Note that I have not used the "effective" optical depth even though the inner parts of the flow are Thomson thick. This is justified by the relativistic nature of the problem. A photon that is emitted at a radius $r$ that corresponds to an optical depth $\tau \gg 1$, does not undergo $\sim \tau^{2}$ scatterings until it escapes as it would be expected from random walk arguments. It suffers $\sim \tau$ scatterings since it preferentially scatters along the radial direction (with an typical angle $\theta \sim 1 / \Gamma \ll 1$ with respect to the radial direction) in the central engine frame.

For a thermal plasma and for characteristic comoving photon energy $h v \ll k_{\mathrm{B}} T_{\mathrm{e}}$, the absorption coefficient is related to the emission $j_{v}$ through the well known expression $\alpha_{v}=$ $j_{v} / 2 v^{2} m_{\mathrm{e}} \Theta_{\mathrm{e}}$. For the mildly relativistic plasma under consideration, the synchrotron emission is well approximated by Eq. (13) of Wardziński \& Zdziarski (2000; see also Petrosian 1981; Petrosian \& McTiernan 1983). Using their expressions for synchrotron emission, I have calculated the turnover frequency $v_{\mathrm{t}}$, defined by the expression $\tau_{v_{\mathrm{t}}}=1$ at different radii in the flow and have verified that $v_{\mathrm{t}} \gg v_{\mathrm{c}}$ (where $v_{\mathrm{c}}=e B^{\prime} / 2 \pi m_{\mathrm{e}} c$ is the cyclotron frequency) for a large range of the model parameters and up to radii that correspond to low optical depths $\sim 0.01$ (or corresponding radii given by Eq. (5)); sufficient for this study. Below $v_{\mathrm{t}}$ the synchrotron emission is strongly self-absorbed, resulting in synchrotron cooling rates in the plasma orders of magnitude less than the Compton cooling rates.

As the analytic estimate (12) shows, the electron temperature increases rather fast and can upscatter the photon field causing deviations from thermal spectra at a radius of about a factor of ten below the Thomson photosphere. On the other hand, this estimate has its limitations since it gives the electron temperature by balancing the electron heating of the electrons to the Compton cooling rate assuming thermal distribution of photons. A more accurate calculation involves a self consistent determination of the electron temperature simultaneously with the actual distribution of the photon field. This calculation is the topic of the next section.

\section{Numerical study}

To study the emergent spectrum and the electron temperature above the "equilibrium" radius in detail, a Monte Carlo code has been developed. It simulates the Compton scattering in a flow with density and bulk Lorentz factor given by Eqs. (1) and (4) respectively, by following the scattering random walk of a large number of photons (Pozdniakov et al. 1983). The special relativistic effects related to both the bulk motion and the scattering cross section of photons in the flow are taken into account. The inner boundary of the computational domain is taken at the last radius where the electrons are in equilibrium with the radiation field, i.e. the equilibrium radius defined by Eq. (13). At this

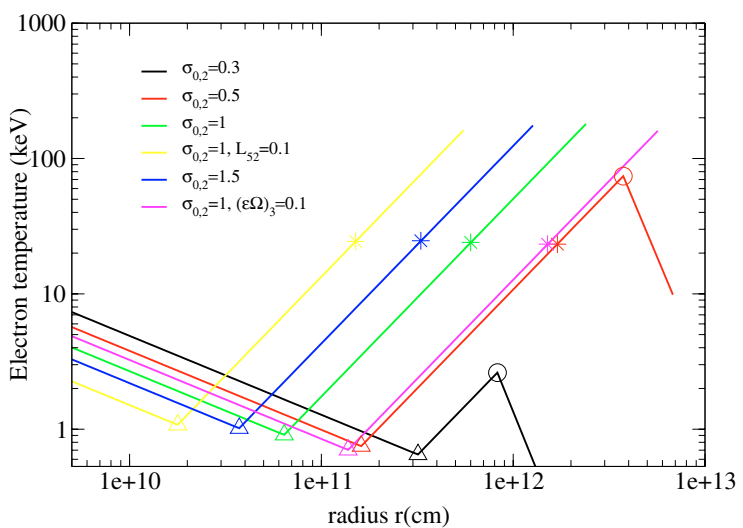

Fig. 1. The comoving electron temperature as a function of radius from the numerically determined energy balance with the radiation field. The baryon loading of the flow is given in the legend. The parameters that are not given are taken to have their reference values (see Sect. 2.1). For small radii radiation and the electrons are in thermal equilibrium. Triangles mark the radius where radiation and electron temperature get out of equilibrium, asterisks mark the photospheric radius and circles mark the saturation radius. Note that the electron temperature at the photosphere is $\sim 25 \mathrm{keV}$ for all the parameter range, except from the high baryon loading (or low $\sigma_{0}$ ) cases.

radius a black body photon spectrum is injected with comoving temperature given by Eq. (9) evaluated at $r_{\text {eq }}$ (given by Eq. (13))

$T_{\text {eq }}=0.9 \frac{f_{\mathrm{e}, 1}^{7 / 27}(\varepsilon \Omega)_{3}^{1 / 9} \sigma_{0,2}^{5 / 18}}{L_{52}^{2 / 27}} \mathrm{keV}$.

The outer boundary of the computational domain is at $r_{\text {out }}$ where the optical depth becomes small (the value $\tau=0.1$ is used).

Obviously the result of the Comptonization depends critically on the electron temperature which, in turn, depends on the Compton cooling rate. The accurate determination of $T_{\mathrm{e}}(r)$ demands an iterative method where the flow is divided in a large number of spherical shells (of the order of $10^{2}$ ) and an initial guess for $T_{\mathrm{e}}(r)$ (e.g. the analytic estimate of Eq. (12)) is given. Then $T_{\mathrm{e}}$ is varied in the different shells until balance of the heating and cooling rates is achieved at all radii. This method is, however, rather computationally demanding. Fortunately, it turns out that one can get very accurate results assuming a power-law dependence of the electron temperature with radius of the form

$T_{\mathrm{e}}=T_{0}\left(\frac{r}{r_{\mathrm{eq}}}\right)^{s}$,

where $T_{0}$ and $s$ are two free parameters to iterate so that heating approximately balances cooling at every radius. The results of such iteration and the emitted photospheric spectra and their energetics for various values of the parameters of the model are the subject of the next section.

\subsection{Results}

The first step toward calculating the photospheric spectra is to determine the electron temperature parameters $T_{0}$ and $s$ for which Compton cooling balances the heating rate of the electrons at every radius in the flow. A first guess for $T_{0}$ and $s$ comes from the analytic estimate (12).

In Fig. 1, I plot the resulting electron temperature as a function of radius for different values of the parameters. At small 


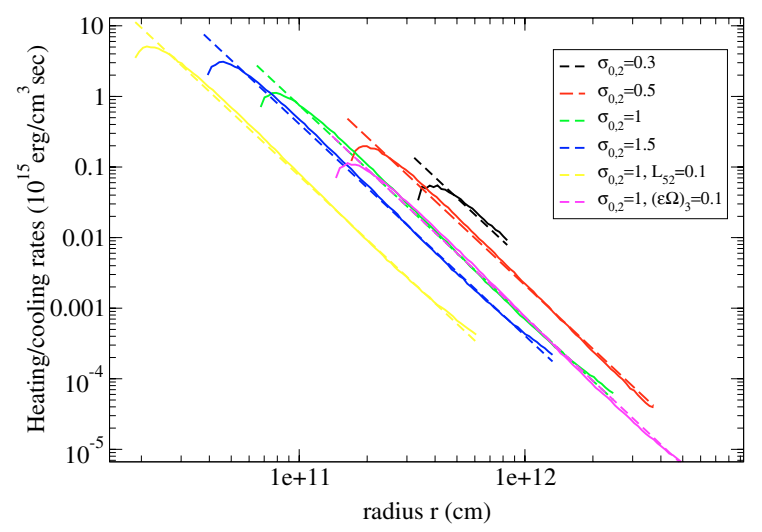

Fig. 2. The heating and cooling rates, dashed and solid lines respectively, as a function of radius for different parameters of the flow given in the legend. The rest of the parameters are assumed to have their reference values (see Sect. 2.1). The cooling and heating rates balance each other well, except from a narrow region close to the "equilibrium" radius in the inner boundary of the computation domain. This illustrates that a power-law is a good approximation for the dependence of the electron temperature with radius.

radii, radiation and matter are in thermal equilibrium. Above the equilibrium radius introduced in Eq. (13), the electron temperature increases following the expression (16) for the numerically computed parameters $T_{0}$ and $s$ which are close to the analytic estimate $T_{\text {eq }}$ and 5/3 respectively. The asterisks in Fig. 1 show the location of the Thomson photosphere. Note that the temperature at the location of the photosphere is rather independent on the parameters of the model and about $\sim 25 \mathrm{keV}$, slightly lower than the analytical estimate (12). For the high $\sigma_{0}$ cases, the electron temperature keeps increasing until the outer boundary of the simulation. For moderate and high $\sigma_{0}$, the temperature is $\sim 200 \mathrm{keV}$ at this outer boundary. The very similar temperatures at the same optical depths in the flow lead to similar $y$ Comptonization parameter and, therefore, similar emitted spectra for a large range of the parameters of the model. This point becomes clear further down in this section where the computed spectra are presented.

Note that in the low $\sigma_{0}$ (or large baryon loading) cases, there is a point above which the electron temperature drops again as a function of radius. This point is the saturation radius $r_{\mathrm{s}}$ (defined by Eq. (4) and marked by circles in Fig. 1). Above this radius no significant dissipation of magnetic energy takes place and the heating rate of the electrons drops quickly. The region $r>r_{\mathrm{s}}$ does not contribute much to the emergent spectra (except from some degree of adiabatic cooling) since the electrons become essentially cold.

In Fig. 2, I show the cooling and heating rate as a function of radius for the numerically iterated values of $T_{0}$ and $s$. Note that the power law modeling for the radial dependence of the electron temperature (see Eq. (16)) leads to a very good balance except in the very inner region of the computed domain. So the modeling of $T_{\mathrm{e}}$ appears to be accurate enough for the purpose of this calculation. Obviously, a smoother fitting function could be used around the equilibrium radius, which would give a better description in this region. However, since the transition region is rather narrow, only a small fraction of the energy is dissipated there. This simplification is not expected to introduce large errors to the calculations and more detailed modeling of the transition has not been pursued.

The emerging spectra from our calculations are given in Fig. 3 in $E \cdot f(E)$ representation for different values of the

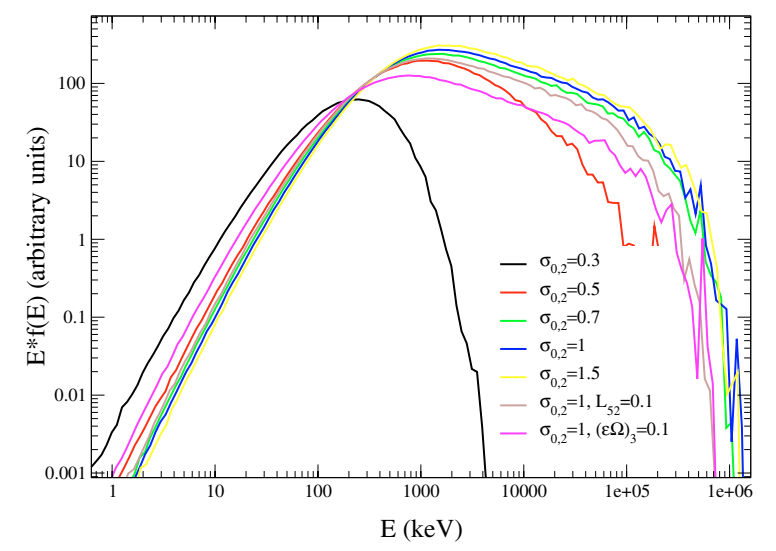

Fig. 3. The resulting $E \cdot f(E)$ spectrum for different values of the parameters of the flow (photon energies in the central engine frame). The moderate and high $\sigma_{0}$ cases exhibit similar spectra with a peak that clusters at $\sim 1 \mathrm{MeV}$ and a flat high energy tail that is a result of unsaturated Comptonization near the photospheric radius. For low $\sigma_{0}$ dissipation stops below the photosphere and the resulting spectrum is quasi-thermal.

parameters of the model. The spectra are plotted in the central engine frame with arbitrary normalization (the energetics are discussed in the next paragraphs). The peak of the spectrum is close to $1 \mathrm{MeV}$ for a large region of the parameter space which is relevant for GRB flows. The clustering of the peak around $1 \mathrm{MeV}$ can be understood by the following considerations. The temperature of the photons at the equilibrium radius in the central engine frame is

$T_{\mathrm{eq}}^{\mathrm{ce}}=\frac{4}{3} \Gamma\left(r_{\mathrm{eq}}\right) T_{\mathrm{eq}}=150 f_{\mathrm{e}, 1}^{1 / 9} L_{52}^{1 / 9}(\varepsilon \Omega)_{3}^{1 / 3} \sigma_{0,2}^{1 / 3} \mathrm{keV}$,

where in the last step the expressions (15), (4) and (13) have been used $^{1}$. The photon spectrum at $r_{\text {eq }}$ peaks at $\sim 4 T_{\text {eq }}^{\mathrm{ce}} \sim 600 \mathrm{keV}$ (in a $E \cdot f(E)$ representation) for the reference values of the parameters and depends very weakly on the flow parameters (see Eq. (17)). The inverse Compton scattering that takes place above the equilibrium radius leads to a moderate increase of the energy of the peak and, most important, to a power-law high energy emission with photon number index $\sim-2.3$ that extends up to a few hundred MeV. This high energy part of the spectrum is the result of unsaturated Comptonization taking place close to the Thomson photosphere and appears for flows with $\sigma_{0} \gtrsim 50$.

Both the peak and the high energy slope lie well within the observed range of the typical GRB spectrum, confirming that the photospheric components may be responsible for the prompt GRB emission. The low energy slope of the spectrum depends on the energy range over which one attempts to fit it. Far from the peak, its slope is about $f(E) \propto E^{2}$ (i.e. with photon number index $\alpha \sim 1$ ). When fitted in the BATSE range (assuming a burst at $z=2$ ) with the Band spectrum (Band et al. 1993), typical values of $\alpha \sim-0.3$ are found for the spectral slope below the peak frequency. These values are compatible with those measured in hard bursts and cannot be explained with the simplest synchrotron models.

In rather low $\sigma_{0} \lesssim 50$ flows, the emerging spectrum has much weaker emission above its peak. In these cases the magnetic dissipation stops close to, or even below, the Thomson

${ }^{1}$ Note that this expression for the photon temperature does not apply for the very low $\sigma_{0} \lesssim 25-30$ cases for which dissipation does not proceed up to $r_{\mathrm{eq}}$ which is the condition for the expressions (9) and, therefore, (15) and (17) to be applicable. 
photosphere and there is only weak Compton upscattering taking place in the photospheric region. Quasi-thermal emission has been observed in a fraction of GRBs (Ryde 2004, 2005) and they may be a result of low $\sigma_{0}$ (i.e. high baryon loading). For even smaller values of $\sigma_{0} \lesssim 25-30$, the magnetic dissipation stops bellow the equilibrium radius and the photon field suffers substantial adiabatic cooling before it decouples from matter. This results in quasi-thermal photospheric spectrum that peaks at tens of $\mathrm{keV}$ or lower.

The X-ray flashes are events that have spectral properties very similar to these of the classical GRBs but with spectral peak below $\sim 50 \mathrm{keV}$ and are believed to belong to the same family with GRBs (e.g. Barraud et al. 2003). It is tempting to identify the X-ray flashes with these very low $\sigma_{0}$ flows. On the other hand, the spectrum of the X-ray flashes is similar to that of the classical GRBs which makes this identification unlikely. It appears more natural, in the context of this model, that $\mathrm{X}$-ray flashes are typical GRB flows viewed off-axis (and therefore with low $\left.E_{\text {peak }}\right)$. Although the issue on the nature of the $\mathrm{X}$-ray flashes is not settled, afterglow modeling seems to support this interpretation (e.g. Granot et al. 2005).

\subsection{Efficiency of the process}

A question quite relevant to the observational relevance of the photospheric component is its strength. A convenient quantity to measure the strength of this component is the photospheric efficiency $e_{\mathrm{ph}}$ defined here as the ratio of the photospheric luminosity to the total luminosity of the flow. In Fig. 4, I plot the $e_{\mathrm{ph}}$ for various $\sigma_{0}$. For large $\sigma_{0}$, the efficiency is rather low $\lesssim 10 \%$ since most of the magnetic energy is dissipated further out in the flow. For moderate values of $\sigma_{0,2} \sim 0.5$, the photospheric component becomes stronger with $e_{\mathrm{ph}} \gtrsim 15 \%$, while its strength is reduced again for low $\sigma_{0}$. In this case, the dissipation stops when the flow is still Thomson thick and radiation is cooled adiabatically before it decouples from matter. These numbers correspond to $L_{52}=1,(\varepsilon \Omega)_{3}=1$. Higher flow luminosities and values of $\varepsilon \Omega$ lead to higher efficiencies and vice versa (see Fig. 4).

In Fig. 4, the "photospheric efficiency" in the BATSE range $e_{\mathrm{ph}, B}$ is also plotted. The BATSE range is taken to be $(30-2000) \mathrm{keV}$ which correspond to the $(30-2000) \times(1+z) \mathrm{keV}$ energy range in the central engine frame. For the redshift, I take $z=2$. In most of the parameter space explored, more than $\sim 60 \%$ of the photospheric emission is in the BATSE range; resulting in $e_{\mathrm{ph}, \mathrm{B}}$ close to $e_{\mathrm{ph}}$ (see Fig. 4).

In the high $\sigma_{0}$ cases, additional radiation is expected to come from outside the computed domain since magnetic dissipation continues up to $r_{\mathrm{s}} \gg r_{\mathrm{ph}}$. In this Thomson thin region, various of the assumptions underlying our calculations may not hold anymore. I have assumed a thermal distribution of the electrons which can no longer be justified outside the photosphere (see next section). Furthermore, the electron temperature increases rapidly with radius. At some point it approaches that of the rest mass of electrons and pair creation should also be taken into account. Last, the non-thermal electron distributions are expected to lead to efficient synchrotron emission and synchrotron cooling cannot be neglected anymore. The problem of radiative transfer in this Thomson thin region depends critically on the particle acceleration process in the regions where magnetic reconnection takes place. Some estimates on the processes at work in this region can be found in the Giannios \& Spruit (2005).

I discuss the issues of the thermalization of the electron distribution and of pair creation more quantitatively in the next subsections.

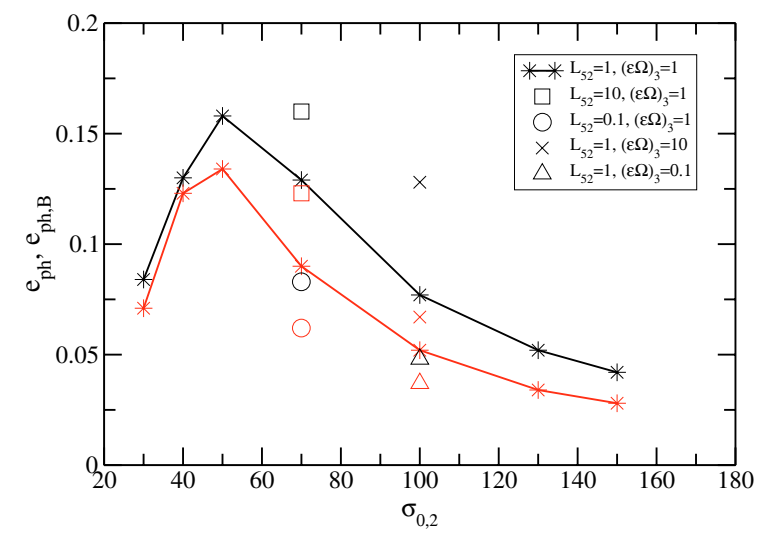

Fig. 4. The ratio of the photospheric luminosity in the whole energy range (black symbols) and in the BATSE energy range (red symbols) to the flow luminosity. Overall the photospheric radiative efficiency varies in the range a few to $20 \%$. Higher flow luminosities and values of $\varepsilon \Omega$ increase the radiative efficiency and vice versa.

\subsection{Thermalization of the electrons}

In our study, I assumed a thermal distribution for the electrons. Particle acceleration in the current sheet in the reconnection regions can lead to highly non-thermal particle distributions but, if the time scale of energy exchange through Coulomb collisions is shorter than the cooling timescale, thermalization of the electron distribution is achieved. The relaxation timescale for the electrons is given by (Spitzer 1967; Stepney 1983)

$t_{\mathrm{ee}}=\frac{4 \sqrt{\pi}}{\ln \Lambda} \frac{\Theta_{\mathrm{e}}^{3 / 2}}{n^{\prime} \sigma_{\mathrm{T}} c}=4 \times 10^{-8} r_{11}^{29 / 6} \frac{f_{\mathrm{e}, 1}^{3 / 2}(\varepsilon \Omega)_{3}^{4 / 3} \sigma_{0,2}^{23 / 4}}{L_{52}^{5 / 2}} \mathrm{~s}$,

where $\ln \Lambda$ is the Coulomb logarithm. For the typical densities and temperatures of the problem at hand the value $\ln \Lambda=13$ is used. I have also used the Eq. (1) and the estimate (12) for the electron temperature (the use of the more accurate numerically calculated values for $T_{\mathrm{e}}$ leads to very similar results).

The cooling timescale of the electrons is

$t_{\text {cool }}=\frac{\frac{3}{2} n^{\prime} k_{\mathrm{B}} T_{\mathrm{e}}}{P_{\mathrm{Comp}}}=5 \times 10^{-7} r_{11}^{7 / 3} \frac{(\varepsilon \Omega)_{3}^{1 / 3} \sigma_{0,2}^{2}}{L_{52}} \mathrm{~s}$,

where the Eqs. (11), (12), (9) and (1) are used in the last step. So, at radius of about $10^{11} \mathrm{~cm}$ the relaxation timescale due to Coulomb collisions is shorter than the cooling timescale. However, the former has steeper radial dependence and the two timescales become equal at a radius $r *$

$r *_{11}=3 \frac{L_{52}^{3 / 5}}{f_{\mathrm{e}, 1}^{2 / 5}(\varepsilon \Omega)_{3}^{2 / 5} \sigma_{0,2}^{3 / 2}}$.

This radius is a factor of 2 inside the photospheric radius for $f_{\mathrm{e}} \simeq$ 1 (compare the last expression with Eq. (6)). Thermalization of the electron distribution is therefore a fair approximation inside the photosphere. Above the location of the photosphere, deviations from thermal distribution are expected. The actual electron distribution, however, is still expected (from energetic considerations) to peak at energies where heating and cooling rates balance each other.

\subsection{Pair creation}

In both the analytical estimates and the numerical investigation, I have neglected pair creation. This is justified close to the 
equilibrium radius where the photon field is thermal with comoving temperature of about $1 \mathrm{keV}$; i.e. much below the pair creation threshold (see minimum of the curves in Fig. 1). However, the electron temperature increases further out in the flow, reaching values of the order of $200 \mathrm{keV}$ near the end of our computational domain at $\tau=0.1$. A fraction of the photons are expected to be upscattered above the pair creation threshold in the comoving frame and lead to pair creation. To estimate how efficiently pair creation takes place, one can define the "comoving compactness" $l^{\prime}=n_{\gamma} \sigma_{\mathrm{T}} r / \Gamma$, where $n_{\gamma}$ is the number density of photons that exceed the rest mass energy of the electrons (in the comoving frame). It can be shown (Lightman 1982; Svensson 1982; Pe'er \& Waxman 2004) that if $l^{\prime}$ is much larger than unity, extensive pair creation takes place in the flow and the number of pairs can exceed that of the electrons related to baryons. On the other hand, if $l^{\prime} \lesssim 1$ pair creation is not substantial.

I have calculated $n_{\gamma}$ directly from our Monte Carlo simulations at different radii and for different values of the parameters of the model. As expected, $l^{\prime}$ is found to increase with radius and to reach values up to $l^{\prime} \lesssim 0.2$ for a large parameter space investigated: $0.3 \leq \sigma_{0,2} \leq 1.5,0.01 \leq L_{52} \leq 10,0.1 \leq(\varepsilon \Omega)_{3} \leq 10$, $0 \leq f_{\mathrm{e}} \leq 1$. So the effect of pair creation is not expected to be important for parameters relevant for GRB outflows in the photospheric region under consideration.

This result can also be understood in view of Fig. 3. The high energy part of the spectrum shows an exponential cutoff at $E_{\mathrm{c}} \sim$ $3 \times 10^{5} \mathrm{keV}$. In the comoving frame, this feature appears at an energy $E_{\mathrm{c}} / \Gamma \sim m_{\mathrm{e}} c^{2}$. So only a small fraction (typically $10^{-3}$ ) of the photospheric luminosity is above the pair creation threshold, resulting in rather low compactness to pair creation.

\section{Discussion}

In this work, I have investigated the appearance of the photospheric component in Poynting-flux dominated GRB outflows in which the magnetic field changes polarity over small scales (AC model). An important characteristic of the model is that it predicts gradual dissipation of magnetic energy through reconnection over many decades of radii including the region where the flow has Thomson optical depths of order unity (Drenkhahn 2002; Drenkhahn \& Spruit 2002).

Here I have shown that, if a large fraction of the energy is dissipated directly to the electrons, the electron temperature increases rapidly at a wide region around the Thomson photosphere. The electron temperature is self-consistently calculated at different radii by balancing the heating and cooling rates. For this calculation both analytical estimates and Monte Carlo simulations are used. It is shown that inverse Compton scattering of the underlying thermal radiation leads to spectra that peak in the $1 \mathrm{MeV}$ range (in the central engine frame) and have power-law high energy part for a wide range of the model parameters. The high energy power law is a result is unsaturated Comptonization that takes place at optical depths of order of unity. When fitted with the "Band" spectrum (Band et al. 1993), the spectra have low and high frequency spectral slopes and peak frequency in agreement with observations. This "photospheric" component is a significant fraction (from $\sim 3 \%$ to more than $15 \%$ ) of the luminosity of the flow and may, therefore, be responsible for the prompt GRB emission.

For high baryon loadings (low $\sigma_{0}$ ) in the flow, the energy dissipation stops below the Thomson photosphere and no spectral component appears above the thermal peak. In this case, the photospheric emission is quasi-thermal and may be responsible for the appearance of a fraction of GRBs (Ryde 2004, 2005).
Our calculation is limited to a region that extends up to a factor of $\sim$ several above the photosphere (where the Thomson optical depth drops to $\tau \sim 0.1$ ). However, models with sufficiently low baryon loading (or equivalently high $\sigma_{0}$ ) predict energy dissipation that continues further out in the flow. The spectra expected by these outer parts of the flow have been to some extent investigated by Giannios \& Spruit (2005). On the other hand, as long as the issue of particle acceleration in the reconnection regions is poorly understood, definite predictions on the relative importance of radiative mechanisms (e.g. Compton scattering, synchrotron emission) or the emitted spectra are hard to be made about the Thomson thin part of the flow.

Our study was limited to the "AC" model because it makes robust predictions concerning the dynamics of the flow and the rate of energy density dissipation. Furthermore, these predictions can take the form of simple algebraic expressions that simplify our study of radiative transfer in the flow. On the other hand, a large variety of models have or may have significant energy dissipation in the photospheric region that can lead to strong deviations from quasi-thermal spectra. In the context of the internal shock model, for example, shocks can also take place close to the location of the photosphere, leading to modifications of the photospheric emission (Mészáros \& Rees 2000; Ryde 2004; Rees \& Mészáros 2005; Pe'er et al. 2005). Another example of dissipative models is that of a strongly magnetized flow with an axisymmetric magnetic field. Such flow is subject to current driven instabilities (kink instability) that lead to gradual dissipation of magnetic energy and rather strong photospheric emission (Giannios \& Spruit 2006).

\section{Conclusions}

The standard fireball model for GRB flows predicts a rather strong photospheric component that is emitted when radiation and matter decouple. A photospheric component is also expected in magnetic GRB models (Drenkhahn \& Spruit 2002; Giannios $\&$ Spruit 2006). In the absence of dissipative processes close to the photosphere, the photospheric component is expected to be quasi-thermal. In that case, a thermal peak should be systematically observed in the prompt GRB spectra, which is not the case (Daigne \& Mochkovitch 2002). On the other hand, most of the GRB models for the prompt emission invoke energy dissipation at a large range of radii. If much energy dissipation takes place in the region of the photosphere, it can lead to large deviation of the photospheric component from purely thermal. Such investigation has been made in the context of internal shock and slow dissipation models by Pe'er et al. (2005).

In this work, I have focused on the photospheric emission expected from a strongly magnetized outflow in which the magnetic field changes polarity over small scales, reconnects and accelerates the flow gradually (Drenkhahn 2002; Drenkhahn \& Spruit 2002). Assuming that a large fraction of the dissipated magnetic energy heats the electrons in the flow, both analytical estimates and numerical calculations show that at a radius that lies a factor of ten below the Thomson photosphere $r_{\mathrm{ph}}$ radiation and matter are no-longer is thermal equilibrium. As a result of the energy dissipation, the flow develops a "hot" photosphere where electron temperature increases as a function of distance with comoving temperatures $\sim \mathrm{a}$ few tens $\mathrm{keV}$ at the location of the photosphere. This result is rather independent of the model parameters such as the luminosity of the flow or the baryon loading $\sigma_{0}$.

Inverse Compton scattering plays an important role at the photosphere, leading to Comptonization spectra that have 
characteristic non-thermal appearance. Fits of the numerically calculated spectra with the Band spectrum (Band et al. 1993) give parameters of the low/high frequency slope and the $E_{\text {peak }}$ of the spectrum in $E \cdot f(E)$ representation in agreement with observations. Furthermore, the observed clustering of the $E_{\text {peak }}$ in the sub-MeV range is a natural outcome of the model. The strength of the photospheric component is $\sim 3-20 \%$ that of the luminosity of the flow and has most $(z 60 \%)$ of its energy in the BATSE energy range. I, therefore, conclude that the photospheric component expected from magnetic models can to a large extent be responsible for the prompt GRB emission.

Acknowledgements. I thank Henk Spruit for many valuable suggestions and discussions and for carefully reading the manuscript. I acknowledge support from the EU FP5 Research Training Network "Gamma Ray Bursts: An Enigma and a Tool."

\section{References}

Abramowicz, M. A., Novikov, I. D., \& Paczyński, B. 1991, ApJ, 369, 175 Band, D., Matteson, J., Ford, L., et al. 1993, ApJ, 413, 281

Barraud, C., Olive, J.-F., Lestrade, J. P., et al. 2003, A\&A, 400, 1021 Coroniti, F. V. 1990, ApJ, 349, 538

Crider, A., Liang, E. P., Smith, I. A., et al. 1997, ApJ, 479, L39

Daigne, F., \& Mochkovitch, R. 2002, MNRAS, 336, 1271

Drenkhahn, G. 2002, A\&A, 387, 714

Drenkhahn, G., \& Spruit, H. C. 2002, A\&A, 391, 1141

Frontera, F., Amati, L., Costa, E., et al. 2000, ApJS, 127, 59

Ghirlanda, G., Celotti, A., \& Ghisellini, G. 2003, A\&A, 406, 879

Ghisellini, G., \& Celotti, A. 1999, A\&AS, 138, 527

Giannios, D., \& Spruit, H. C. 2005, A\&A, 430, 1
Giannios, D., \& Spruit, H. C. 2006, A\&A, 450, 887

Goodman, J. 1986, ApJ, 308, L47

Granot, J., Ramirez-Ruiz, E., \& Perna, R. 2005, ApJ, 630, 1003

Liang, E. P. 1997, ApJ, 491, L15

Lightman, A. P. 1982, ApJ, 253, 842

Lyubarsky, Y. E. 2005, MNRAS, 358, 113

Lyutikov, M., \& Uzdensky, D. 2003, ApJ, 589, 893

Lyutikov, M., \& Blandford, R. D. 2003 [arXiv:astro-ph/0312347]

Mészáros, P., \& Rees, M. J. 1997, ApJ, 482, L29

Mészáros, P., \& Rees, M. J. 2000, ApJ, 530, 292

Paczyński, B. 1986, ApJ, 308, L43

Pe'er, A., \& Waxman, E. 2004, ApJ, 613, 448

Pe'er, A., Mészáros, P., \& Rees, M. J. 2005, ApJ, 635, 476

Pe'er, A., Mészáros, P., \& Rees, M. J. 2006, ApJ, 642, 995

Petrosian, V. 1981, ApJ, 251, 727

Petrosian, V., \& McTiernan, J. M. 1983, Phys. Fluids, 26, 3023

Piran, T. 1999, Phys. Rep., 314, 575

Pozdnyakov, L. A., Sobol, I. M., \& Sunyaev, R. A. 1983, Astrophys. Space Phys. Rev., 2, 189

Preece, R. D., Briggs, M. S., Mallozzi, R. S, et al. 1998, ApJ, 506, L23

Ramirez-Ruiz, E. 2005, MNRAS, 363, L61

Rees, M. J., \& Mészáros, P. 1994, ApJ, 430, L93

Rees, M. J., \& Mészáros, P. 2005, ApJ, 628, 847

Rybicki, G. B. , \& Lightman, A. P. 1979, Radiative Processes in Astrophysics (New York: Wiley)

Ryde, F. 2004, ApJ, 614, 827

Ryde, F. 2005, ApJ, 625, L95

Sari, R., \& Piran, T. 1997, MNRAS, 287, 110

Spitzer, L. 1956, Physics of Fully Ionized Gases (New York: Wiley)

Spruit, H. C., Daigne, F., \& Drenkhahn, G. 2001, A\&A, 369, 694

Stepney, S. 1983, MNRAS, 202, 467

Svensson, R. 1982, ApJ, 258, 335

Thompson, C. 1994, MNRAS, 270, 480

Vlahakis, N., \& Königl, A. 2003, ApJ, 596, 1104

Wardziński, G., \& Zdziarski, A. A. 2000, MNRAS, 314, 183 